\title{
ULTRABRIGHT SYNCHROTRON RADIATION OPENS UP NEW RESEARCH FRONTIERS
}

\author{
G. Margaritondo
}

\author{
Institut de Physique Appliquée, Centre de Spectromicroscopie \\ Ecole Polytechnique Fédérale, 1015 Lausanne, Switzerland
}

\begin{abstract}
We present an update on recent events concerning ultrabright synchrotron radiation. First and foremost, a new generation of sources has been put in operation in the past few months. Second, microscopy and spectromicroscopy - arguably the techniques that will mostly profit from ultrahigh brightness - have produced interesting results demonstrating that they are absolutely needed for a realistic picture of solid surfaces and interfaces, as well as for biological applications. Third, ultrahigh-resolution photoemission has broken new ground in the study of collective phenomena, notably the properties of the superconducting gap. Finally, we will comment on the recent use of another class of sources for practical research: infrared free electron lasers.
\end{abstract}

PACS numbers: $79.60 .-\mathrm{i}, 07.85 .+\mathrm{n}$

\section{A rapid evolution}

The last time I had the honor to lecture at the International School of Synchrotron Radiation in Jaszowiec, I argued that we were on the threshold of a real revolution in synchrotron science. A new generation - the third - of synchrotron sources was in the final stages of construction. And many groups were already involved in the preliminary versions of the techniques that will probably explode with the new sources, notably spectroscopy with high lateral resolution - the so-called spectromicroscopy [1].

I am now happy to report that the expected revolution is happening: I present an update on the new sources and the new classes of experiments.

In the course of 1993, three soft-X-ray sources of the third generation have been put in operation (in addition to the hard-X-ray source ESRF in Grenoble): ALS - Berkeley, ELETTRA - Trieste and SRRC - Taiwan. Furthermore, we have seen the steady user operation of the free electron lasers (FEL's) at Vanderbilt University and at the University of California-Santa Barbara, and the progress towards a similar status of the FEL's facilities of FOM in Holland and of LURE in France.

All of these events are symbolized by Fig. 1, which shows the very first experimental results produced by ELETTRA in Trieste [2]. The bottom part of the 

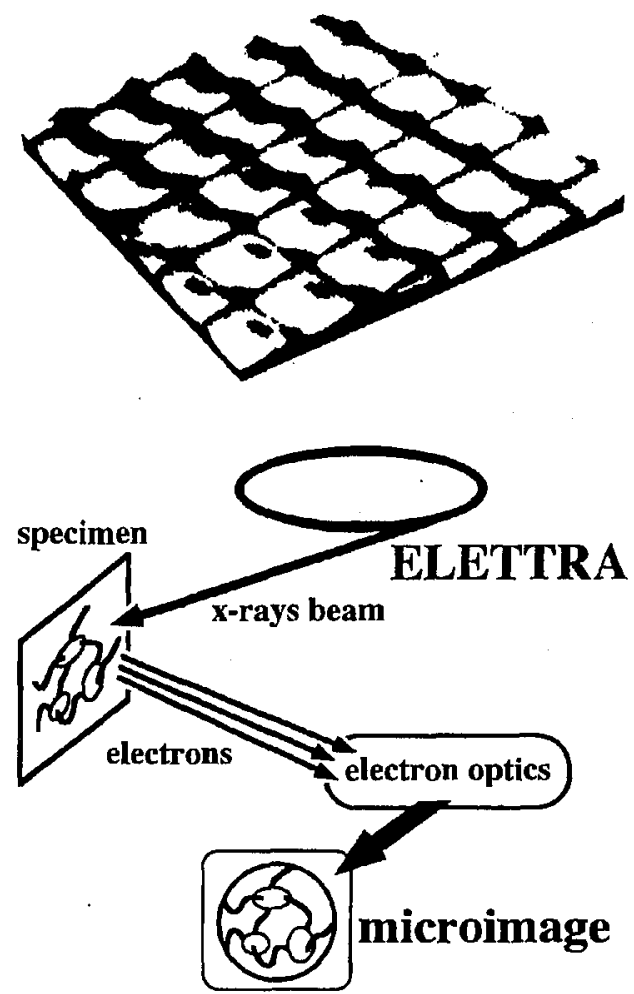

Fig. 1. Top: the first experimental result obtained on ELETTRA: three-dimensional reconstruction (with the @Spyglass software) of the X-ray photoelectron micrograph of a metal mesh (2 $\mu \mathrm{m}$ steps). Data from Ref. [2]. Bottom: artist's view of the experimental system (photoelectron emission microscope or PEEM).

figure illustrates the experiment, in which synchrotron photons create photoelectrons, which in turn are processed to create a micrograph. The top part shows the image of a metal mesh, with approximately 1 micron lateral resolution.

This result crowned a most successful final stage of construction and commissioning, under the leadership of Carlo Rubbia and Giuseppe Viani: an electron beam circulated in ELETTRA hours after the first injection was attempted; the promised beam specifications were quickly reached and surpassed. Within two and a half weeks, a team of scientists from the Sincrotrone Trieste company, from the Istituto di Struttura della Materia and from the Ecole Polytechnique Fédérale de Lausanne were taking the first data [2], of which Fig. 1 is an example.

We would like to take this opportunity to thank all the people that made this amazing result possible, in particular the former leader of ELETTRA's accelerator division, Mario Puglisi, who unfortunately died shortly before the first injection, and his successor Albin Wrülich, together with all of the staff members of the Sincrotrone Trieste company. 
The extremely fast commissioning of ELETTRA was not an isolated event: the commissioning in 1993 of the Advanced Light Source in Berkeley and of the Taiwan Synchrotron Radiation Research Center were also astonishingly fast. All of these sources were commissioned in time, within budget and surpassing their promised performances.

\section{New research opportunities: spectromicroscopy}

The question now is: how can we use these amazing new facilities for what they are worth? What is, in other words, the best use of ultrahigh brightness? There is of course no single answer to that, but we believe that some areas of traditional synchrotron activities will particularly profit from the huge increase in brightness.

Brightness is particularly important for microscopy, and even more important for the combination of synchrotron spectroscopy and microscopy, for example for photoemission spectromicroscopy [1]. Figure 1 shows an example of the use of photoelectrons to create microimages, without, however, performing a spectroscopic analysis. Suppose, however, that either the electron energy or the photon energy are scanned: one can bring in this way the full power of the corresponding spectroscopic techniques to the microscopic domain [1].

The need for lateral resolution in spectroscopies like photoemission has recently been demonstrated by some interesting results. Perhaps the most striking example is photoemission spectromicroscopy of insulators. Coluzza et al. [3] used a SCIENTA ESCA 300 spectromicroscope at the Lausanne's Center of Spectromicroscopy to investigate a number of insulating systems. They found that their core-level photoemission peaks are affected by local charging effects, which cause rigid shifts in energy. The shifts' magnitude dramatically changes from place to place on the scale of $30-300 \mu \mathrm{m}$.

Conventional spatially-integrated photoemission core peaks can, therefore, be the superposition of different components. This affects the apparent peak's line shape, position in energy and spin-orbit branching ratio. The consequences are far-reaching: virtually every photoemission spectrum taken for insulating materials could be affected by these artifacts.

The aforementioned effects are quite evident, for example, in the single-crystal $\mathrm{NaCl} \mathrm{Cl} 2 p$ and $\mathrm{Na} 1 s$ spectra of Fig. 2, taken with a charge-neutralizing flood gun. The three lower sets of spectra concern three different adjacent $40 \times 300 \mu \mathrm{m}$ areas on a straight line (similar results were obtained for spectra from $40 \times 40 \mu \mathrm{m}$ spots). The spatial differences in peak energy position are quite evident. Such differences shifts are equal for the two core levels, suggesting an electrostatic effect, such as, indeed, charging.

The upper curves in each set of Fig. 2 are normalized averages of the spatially-resolved spectra, and roughly simulate the results of spatial integration in a conventional photoemission experiment. Similar phenomena were found [3] for several other insulating systems, specifically: layers of silicon oxide on $\mathrm{Si}(100)$, granular $\mathrm{Sc}_{2} \mathrm{O}_{3}$ pellets, CsI single crystals, zeolite, diamond films, and $\mathrm{Cu}$-containing superconducting oxides.

The causes of the electrostatic shifts' spatial variations are not clear, and 


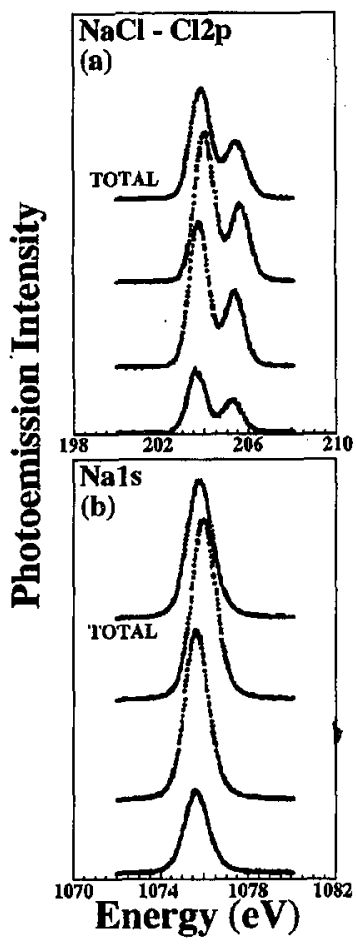

Fig. 2. Spatially- resolved $\mathrm{Al} K_{\boldsymbol{x}}$ photoemission spectra taken on single-crystal $\mathrm{NaCl}$, for (a) the $\mathrm{Cl} 2 p$ and (b) the $\mathrm{Na} 1 s$ core levels. Spectra from three different adjacent $40 \times 300 \mu \mathrm{m}$ areas along a line are shown, together with their normalized sum (simulating the effects of spatial integration). The spectra were taken with a flood gun. Data from Coluzza et al., Ref. [3].

could be different for different samples and/or different experiments. Whatever their causes, the shifts have a dramatic impact on the interpretation of conventional, spatially integrated photoemission spectra of insulators: the superposition of shifted components can affect the general line shape, perhaps simulating asymmetries or an unresolved fine structure, or significantly altering the apparent spin-orbit branching ratio.

This makes it difficult to derive fine chemical information from the line shape analysis of spatially-integrated spectra: the case for spectromicroscopy is, therefore, quite compelling.

Synchrotron photoemission spectromicroscopy has produced a stream of similarly interesting results in the past few months. For example, De Stasio et al. [4] extensively used Brian Tonner's X-ray secondary emission microscope (XSEM) [5] revealing the distribution of toxic elements in neuron cultures. The XSEM is an electron-optics-imaging instrument, in which the spectroscopy is implemented by scanning the photon energy.

Quite interesting results have also been obtained by photon-focusing and 
scanning photoemission spectromicroscopy. The record for lateral resolution is held at present by the Schwarzschild-objective scanning spectromicroscope MAXIMUM [6], developed at Wisconsin by an international group under the leadership of Franco Cerrina. The best achieved resolution is of the order of $900 \AA$. Instruments of this kind are no longer feasibility projects, since they have been systematically used for real experiments. In the case of MAXIMUM, scanning images and small-area spectra were obtained in a variety of systems by A.K. Ray-Chaudhury [6], S. Liang [7], and W. Ng [8].

The recent MAXIMUM data again illustrate the need for high lateral resolution in photoemission experiments. For two decades, photoemission has been a leading tool in the study of solid surfaces and interfaces. But the lack of lateral resolution did not allow it to address the basic question: are the surface and interface properties varying from place to place? The most recent results show that almost always they do:

- Cerrina et al. [9] and independently P. Pianetta and his co-workers [10] discovered that the band bending at cleaved semiconductor surfaces can change from place to place.

- Gozzo et al. [11] found lateral variations in the energy barrier between a gold or germanium overlayer and a GaSe substrate. This indicates that Schottky barriers and heterojunction band lineups may also vary from place to place. And there is no general rule about that, since Pianetta and his co-workers [12] found that $\mathrm{Sb}$ overlayers on $\mathrm{GaAs}(110)$ tend to reduce the lateral variations in band bending.

In summary, photoemission spectromicroscopy is rapidly becoming a well established technique, and a basic tool rather than a luxury. This evolution will be undoubtedly by the new ultrabright synchrotron sources.

\section{Ultrahigh resolution spectroscopy}

High energy and angular resolution is another excellent way to exploit ultrahigh brightness. Photoemission has been performed for decades at resolutions ranging from $100-500 \mathrm{meV}$. It is now routinely possible to work at $10-20 \mathrm{meV}$ or even better.

This means that new classes of phenomena are becoming accessible. In particular, collective phenomena. For example, photoemission has rapidly become the basic tool to investigate the nature of high-temperature superconductivity. This initiated with the mere observation of the superconducting gap in $\mathrm{BiCaSrCuO}$ [13].

Recently, however, high-resolution photoemission has become much more sophisticated, and is used to explore what is arguably the central issue in today's debate about the nature of high-temperature superconductivity: the symmetry of the order parameter.

This issue is the center of a strong controversy, opposing partisans of $s$-waves, $d$-waves and other schemes. The group of Marshall Onellion, in cooperation with our group, has explored this issue, for example by measuring the size of the gap in different directions [13]. 
The result is that the gap is not the same in different directions, which rules out isotropic $s$-waves. But $s$ is not zero along any high-symmetry direction, which is in conflict with the simplest type of $d$-waves. Furthermore, the temperature dependence of the gap is different in different directions.

All of these results suggest a novel approach to the problem, based on a two-component order parameter (as recently suggested by Robert Joynt [14]), or perhaps on anisotropic $s$-waves.

These were not the only contributions of high-resolution photoemission. We note, for example, the discovery and subsequent study of the suppression of the photoemission signal near the Fermi level, which appears as a basic property of almost all of the one-dimensional systems [15].

\section{Conclusions and future evolution}

Synchrotrons are not the only sources that are stimulating and allowing the present revolution. In the complementary infrared spectral domain, FEL's are rapidly becoming essential tools. Figure 3 shows a recent example, obtained by an American-Swiss program at the Vanderbilt University FEL: the two-photon absorption spectra of germanium by Tuncel et al. [16] (revealed by measuring the photoconductivity), in the energy region of the direct and indirect Ge gaps.

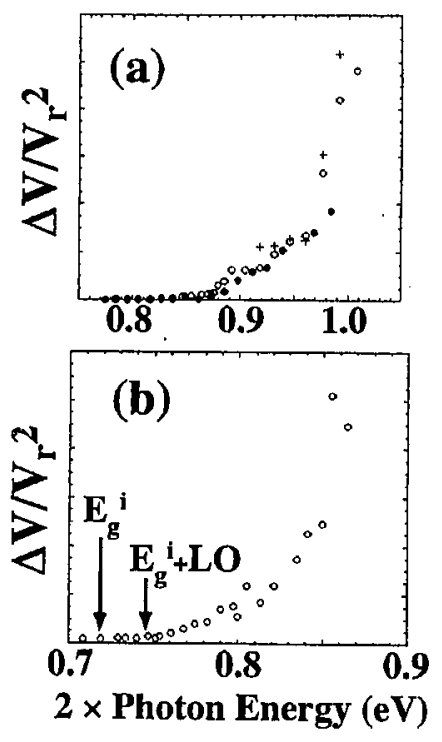

Fig. 3. (a) FEL two-photon-absorption induced photoconductivity signal of germanium (normalized to the square of the intensity reference signal), as a function of double the incident photon energy. We note that direct-gap threshold is corresponding to the gap width of $0.87 \mathrm{eV}$ at $125 \mathrm{~K}$. (b) Similar data for the indirect gap. Data from Tuncel et al., Ref. [16]. The vertical arrows mark the indirect gap width, $E_{\mathrm{g}}^{\mathrm{i}}$, and the corresponding threshold for LO-phonon assisted processes as predicted by Bassani-Hassan theory [17]. 
From the threshold, Tuncel et al. [16] estimated a direct gap width $E_{\mathrm{g}}^{\mathrm{d}}=$ $0.87 \pm 0.01 \mathrm{eV}$; this value is reasonable considering the published value and the estimated sample temperature. The $E_{\mathrm{g}}^{\mathrm{d}}$ value was then used to analyze the indirect gap data, deriving an indirect gap width $E_{\mathrm{g}}^{\mathrm{i}}=0.718 \pm 0.01 \mathrm{eV}$ at $125 \mathrm{~K}$, corresponding to the left-side arrow labeled as $E_{\mathrm{g}}^{\mathrm{i}}$ in the figure.

The actual two-photon indirect-gap threshold is clearly shifted with respect to $E_{\mathrm{g}}^{\mathrm{i}}$, even considering the experimental uncertainty. As stown by the arrow labeled as $E_{\mathrm{g}}^{\mathrm{i}}+\mathrm{LO}$, the shift is consistent with a process assisted by the emission of a $30.4 \mathrm{meV} \mathrm{LO}\left(L_{6^{-}}\right)$phonon. The two-photon indirect threshold is therefore, as predicted by Bassani and Hassan [17] in 1972, primarily an LO-assisted process. Note the long time between the formulation of the Bassani-Hassan theory [17] and the experimental test of Tuncel et al. [16]. The reason is, of course, the weak absorption at the indirect threshold in the case of germanium.

The measurements would have been impossible with conventional lasers, whereas they were quite simple with the FEL. And this is but one example of the many potential applications of the FEL for condensed matter research. The Vanderbilt FEL has also been used by Coluzza et al. and McKinley et al. [18] as a pump to excite carriers over energy barriers at biased semiconductor interfaces. From the corresponding photocurrent thresholds, it is very straightforward to derive the energy barrier heights [18].

All of these examples show that the predicted revolution of 1993-1994 is indeed happening. But a new revolution is already appearing at the horizon. The Paul-Scherrer Institut in Villigen, Switzerland, has just produced the preliminary study of a synchrotron soft-X-ray source (SLS or Swiss Light Source) whose brightness could surpass that of ELETTRA and of the ALS by up to one order of magnitude. We must, therefore, start thinking about even more innovative ways to use the new photon sources which an amazing progress in technology is giving us.

\section{Acknowledgments}

The activities of the Centre de Spectromicroscopie of the Ecole Polytechnique Fédérale de Lausanne (EPFL) are supported by the Fonds National Suisse de la Recherche Scientifique, by the EPFL, by the US Office of Naval Research, by the US National Science Foundation and by the Sincrotrone Trieste SCpA. We are grateful to C. Coluzza, J.-L. Staehli, J. Almeida, Ph. Alméras, J.-P. Baudat, H. Berger, I. Collins, T. dell'Orto, F. Gozzo, H. Jottérand, M. Marsi, R. Sanjinés, A. Terrasi, E. Tuncel, and all our collaborators of the EPFL Centre de Spectromicroscopie for their role in the success of the center. We are equally grateful to our external partners, the members of the groups headed by Marshall Onellion, Franco Cerrina, Gelsomina De Stasio, Delio Mercanti, Brian Tonner, Marc Ilegems and Norman Tolk.

\section{References}

[1] Photoemission: from the Past to the Future, Eds. C. Coluzza, R. Sanjinés, G. Margaritondo, EPFL, Lausanne 1992; G. Margaritondo, F. Cerrina, Nucl. Instrum. Methods. Phys. Res. A 291, 26 (1990); Selected Experiments in Condensed Matter Physics with Synchrotron Radiation, Ed. W. Czaja, Birkhäuser, Basel 1991. 
[2] A. Abrami, S. Antonini, M. Bernardini, M. Bertolo, C.J. Bocchetta, D. Bulfone, F. Cargnello, F. De Bona, M. Giannini, W. Jark, A. Massarotti, M. Puglisi, R. Richter, A. Rindi, R. Rosei, C. Rubbia, A. Savoia, G. Viani, R.P. Walker, Albin Wrülich, C. Coluzza, Tiziana Dell'Orto, F. Gozzo, G. Margaritondo, Gelsomina De Stasio, P. Perfetti, Nucl. Instrum. Methods, in press.

[3] C. Coluzza, J. Almeida, Tiziana dell'Orto, F. Gozzo, P. Alméras, H. Berger, D. Bouvet, M. Dutoit, S. Contarini, G. Margaritondo, unpublished, and the references therein.

[4] Gelsomina De Stasio, D. Dunham, B.P. Tonner, Delio Mercanti, M. Teresa Ciotti, A. Angelini, C. Coluzza, P. Perfetti, G. Margaritondo, Neuroreports 4, 1175 (1993), and the references therein.

[5] B.P. Tonner, Nucl. Instrum. Methods Phys. Res. A 291, 60 (1990).

[6] C. Capasso, W. Ng, A.K. Ray-Chaudhuri, S.H. Liang, R.K. Cole, Z.Y. Guo, J. Wallace, F. Cerrina, J. Underwood, R. Perera, J. Kortright, G. De Stasio, G. Margaritondo, Surf. Sci. 287/88, 1046 (1993); A.K. Ray-Chaudhuri, W. Ng, S. Liang, F. Cerrina et al., unpublished; A.K. Ray-Chaudhuri, Thesis, University of Wisconsin-Madison, 1993.

[7] S. Liang, Thesis, University of Wisconsin-Madison, 1993.

[8] W. Ng, Thesis, University of Wisconsin-Madison, 1993.

[9] F. Cerrina, A.K. Ray-Chaudhuri, W. Ng, S. Liang, S. Singh, J.T. Welnak, J.P. Wallace, C. Capasso, J.H. Underwood, J.B. Kortright, R.C.C. Perera, G. Margaritondo, Appl. Phys. Lett. 63, 63 (1993).

[10] C. Kim, P.L. King, P. Pianetta, J. Vac. Sci. Technol. B 10, 1944 (1992).

[11] F. Gozzo, M. Marsi, H. Berger, G. Margaritondo, A. Ottolenghi, A.K. Ray-Chaudhuri, W. Ng, S. Liang, S. Singh, J.T. Welnak, J.P. Wallace, C. Capasso, F. Cerrina, Phys. Rev. B 48, 17163 (1993); F. Gozzo, H. Berger, I.R. Collins, G. Margaritondo, W. Ng, A.K. Ray-Chaudhuri, S. Liang, S. Singh, F. Cerrina, unpublished.

[12] P. Pianetta, private communication.

[13] Jian Ma, R.J. Kelley, C. Quitmann, H. Berger, G. Margaritondo, M. Onellion, Phys. Rev. $B$, in press, and unpublished, and the references therein.

[14] Robert Joynt, private communication.

[15] Y. Hwu, P. Alméras, M. Marsi, H. Berger, F. Lévy, M. Grioni, D. Malterre, G. Margaritondo, Phys. Rev. (Rapid Commun.) B 46, 13624 (1992); C. Coluzza, H. Berger, P. Alméras, F. Gozzo, G. Margaritondo, G. Indlekofer, L. Forro, Y. Hwu, Phys. Rev. $B$ 47, 6625 (1993).

[16] E. Tuncel, J.L. Staehli, C. Coluzza, G. Margaritondo, J.T. McKinley, X. Yang, N.H. Tolk, Phys. Rev. Lett. 70, 4146 (1993).

[17] F. Bassani, A.R. Hassan, Nuovo Cimento B 7, 313 (1972).

[18] C. Coluzza, E. Tuncel, J.-L. Staehli, P.A. Baudat, G. Margaritondo, J.T. McKinley, A. Ueda, A.V. Barnes, R.G. Albridge, N.H. Tolk, D. Martin, F. Morier-Genoud, C. Dupuy, A. Rudra, M. Ilegems, Phys. Rev. B 46, 12834 (1992); N.H. Tolk, C.A. Brau, G.S. Edwards, G. Margaritondo, J.T. McKinley, Proc. Conf. on Short-Wavelength Radiation Sources, San Diego, California 1991, SPIE Proceedings Series, Vol. 1552, Bellingham, Washington 1991, p. 7. 